\title{
Tracks and Voids in Amorphous Ge Induced by Swift Heavy-Ion Irradiation
}

\author{
M. C. Ridgway, ${ }^{1, *}$ T. Bierschenk, ${ }^{1}$ R. Giulian,,${ }^{1,}$ B. Afra,${ }^{1}$ M. D. Rodriguez, ${ }^{1}$ L. L. Araujo, ${ }^{1, \dagger}$ A. P. Byrne,,${ }^{1, *}$ \\ N. Kirby, ${ }^{2}$ O. H. Pakarinen, ${ }^{3}$ F. Djurabekova, ${ }^{3}$ K. Nordlund,${ }^{3}$ M. Schleberger, ${ }^{4}$ O. Osmani, ${ }^{4,5,8}$ \\ N. Medvedev, ${ }^{5, \|}$ B. Rethfeld, ${ }^{5}$ and P. Kluth ${ }^{1}$ \\ ${ }^{1}$ Research School of Physics and Engineering, Australian National University, Canberra 0200, Australia \\ ${ }^{2}$ Australian Synchrotron, Clayton 3168, Australia \\ ${ }^{3}$ Department of Physics and Helsinki Institute of Physics, University of Helsinki, 00014 Helsinki, Finland \\ ${ }^{4}$ Fakultät für Physik, Universität Duisburg-Essen, 47057 Duisburg, Germany \\ ${ }^{5}$ Department of Physics and OPTIMAS Research Center, Technical University of Kaiserslautern, 67663 Kaiserslautern, Germany
}

(Received 17 January 2013; revised manuscript received 2 May 2013; published 14 June 2013)

\begin{abstract}
Ion tracks formed in amorphous Ge by swift heavy-ion irradiation have been identified with experiment and modeling to yield unambiguous evidence of tracks in an amorphous semiconductor. Their underdense core and overdense shell result from quenched-in radially outward material flow. Following a solid-toliquid phase transformation, the volume contraction necessary to accommodate the high-density molten phase produces voids, potentially the precursors to porosity, along the ion direction. Their bow-tie shape, reproduced by simulation, results from radially inward resolidification.
\end{abstract}

DOI: 10.1103/PhysRevLett.110.245502

PACS numbers: 61.80.Jh, 61.43.Dq, 61.43.Bn, 61.05.cf

Swift heavy-ion irradiation (SHII) has many applications, spanning geochronological dating [1] to nanostructure fabrication [2]. Though this approach has found industrial application [3], the fundamental nature of ionsolid interactions at very high ion energies remains poorly understood. Such interactions are dominated by inelastic processes (electronic stopping) resulting in the excitation and ionization of substrate atoms while, in contrast, the elastic processes (nuclear stopping) that lead to ballistic atomic displacements at much lower energies are negligible in the SHII regime. The efficiency with which energy deposited in the electronic subsystem is subsequently transferred to the lattice is governed by the electronphonon coupling parameter $g$ where typically $g_{\text {amorphous }}>$ $g_{\text {crystalline }}$ due to a reduced electron mean free path in the former. When the lattice temperature exceeds that required for melting, a narrow cylinder of molten material is formed along the ion path. The ensuing rapid resolidification of this transient liquid phase can yield remnant structural modifications within the substrate in the form of an ion track.

Crystalline $\mathrm{Ge}(c-\mathrm{Ge})$ is relatively insensitive to SHII such that ion-track production necessitates very high electronic stopping $S_{e}$ values. Discontinuous tracks follow single-ion irradiation $\left(S_{e}=35 \mathrm{keV} / \mathrm{nm}\right)$ [4,5] while cluster-ion irradiation $\left(S_{e}=37-51 \mathrm{keV} / \mathrm{nm}\right)$ yields tracks of diameter 5-15 nm [5]. In contrast, amorphous $\mathrm{Ge}(a-\mathrm{Ge})$ is rendered porous under SHII with $S_{e}>\sim 10 \mathrm{keV} / \mathrm{nm}$ [6] while ion hammering results for $S_{e}>\sim 12 \mathrm{keV} / \mathrm{nm}$ [6], the latter manifested as a nonzero deformation yield [7]. These observations are consistent with $g_{\text {amorphous }}>$ $g_{\text {crystalline }}$ and ion-track formation has been suggested as the origin of these two phenomena [6,7]. A recent molecular dynamics (MD) study of irradiated $a$-Ge [8] suggested voids originate from outgoing shock waves resulting from rapid heating and expansion of the ion-track core. The sole report of ion tracks in $a-\mathrm{Ge}$ is that of Furuno et al. [9] who reported recrystallization of tracks in a $5-\mathrm{nm} a-\mathrm{Ge}$ layer following SHII in a grazing-incidence orientation, a geometry that can lead to significant reductions in threshold $S_{e}$ values for ion-track formation [10]. The proximity of the surface could also perturb resolidification and enable recrystallization given the molten ion track is no longer completely surrounded by an amorphous matrix.

An unambiguous identification of ion tracks in amorphous semiconductors has significant implications. The pioneering report of Hedler et al. [11] demonstrating a glass transition in amorphous $\mathrm{Si}$ was predicated on iontrack formation yet the latter remains unverified. The lack of direct evidence stems from difficulties in detection. For example, transmission electron microscopy (TEM) identification of amorphous ion tracks in an amorphous matrix is impeded by the lack of diffraction and absorption contrast. In contrast, small-angle $\mathrm{x}$-ray scattering (SAXS) is an effective means of characterizing ion tracks under such conditions, as we have demonstrated for amorphous $\mathrm{SiO}_{2}$ [12]. For this report, we now combine physical characterization using SAXS and TEM with a multi-time-scale theoretical approach including an asymptotical trajectory Monte Carlo (MC) calculation of the electron dynamics, a two-temperature model (TTM) description of the heat dissipation and a MD simulation of the atom dynamics for ion tracks in $a$-Ge. With this novel approach, we characterize ion-track creation and evolution theoretically and ion-track structure experimentally.

An $a$-Ge layer of thickness $2.5 \mu \mathrm{m}$ was formed on crystalline $\mathrm{Si}$ then irradiated with $185-\mathrm{MeV} \mathrm{Au}^{13+}$ ions $\left(S_{e}=\sim 22 \mathrm{keV} / \mathrm{nm}\right.$ [13]). Samples were subsequently 
characterized with SAXS and cross-section TEM (XTEM). For experimental details, see the Supplemental Information [14].

The simulation of ion tracks in $a$-Ge induced by SHII was achieved via three interconnected stages. The first included the excitation of electrons due to the ion energy deposition. The electron dynamics were then followed by combining a MC approach [15] and TTM [16-18]. While the MC method is a kinetic means of modeling the initial electron excitation and the nonequilibrium electron dynamics, the TTM describes the energy transport within the electronic and lattice subsystems. Using the MC TTM approach, the rate of energy transfer between the electronic and lattice subsystems ( $\left.g_{\text {amorphous }}\right)$ was extracted and so fitting this parameter was unnecessary. Instead, the electron-phonon coupling and specific heat capacity were calculated explicitly as a function of electron temperature (see Supplemental Information [14]). Here we do not consider ultrafast phase transformations like athermal melting [19] because the particular time scale of the relevant phase transformation is of minor importance. The calculation of the lattice temperature is used here to judge whether the localized energy is sufficient to induce and maintain the molten state on a time scale of several picoseconds.

The evolution of the lattice temperature is mirrored in the MD simulation where the spatially varying, timedependent energy transfer between the two subsystems from the MC TTM calculation was used as input for continuously depositing kinetic energy in a random direction on the lattice atoms until the two subsystems were in equilibrium at $3 \mathrm{ps}$. MD simulations were performed with the PARCAS code [20] using the Stillinger-Weber (SW) and Tersoff potentials $[21,22]$. The choice of potential was governed by the most accurate description of the solidto-liquid and liquid-to-solid phase transformations. The simulation cell volume was $61 \times 41 \times 41 \mathrm{~nm}^{3}$. Note that MD simulations with fixed cell dimensions (mechanically rigid boundaries) coupled with a volume contraction within the ion track (demonstrated below) could potentially overestimate the resultant tensile stress compared to that for elastically responding boundaries. The initial $a$-Ge cell was constructed from $c$-Ge using the Wooten-WinerWearie (WWW) approach [23] (see Supplemental Information [14]). Using the MC TTM result as input, the molten track width deduced from MD was half that measured experimentally, an extraordinary result given the absence of fitted parameters. To better enable the comparison of calculation, simulation, and experiment, the MC TTM input was then scaled so the width of the molten ion track extracted from MD was consistent with that determined from SAXS.

SAXS detector images [Fig. 1(a) and 1(b) insets] were comprised of two anisotropic scattering components separable by tilting the sample relative to the x-ray direction. The scattering intensity $I$ as a function of the scattering
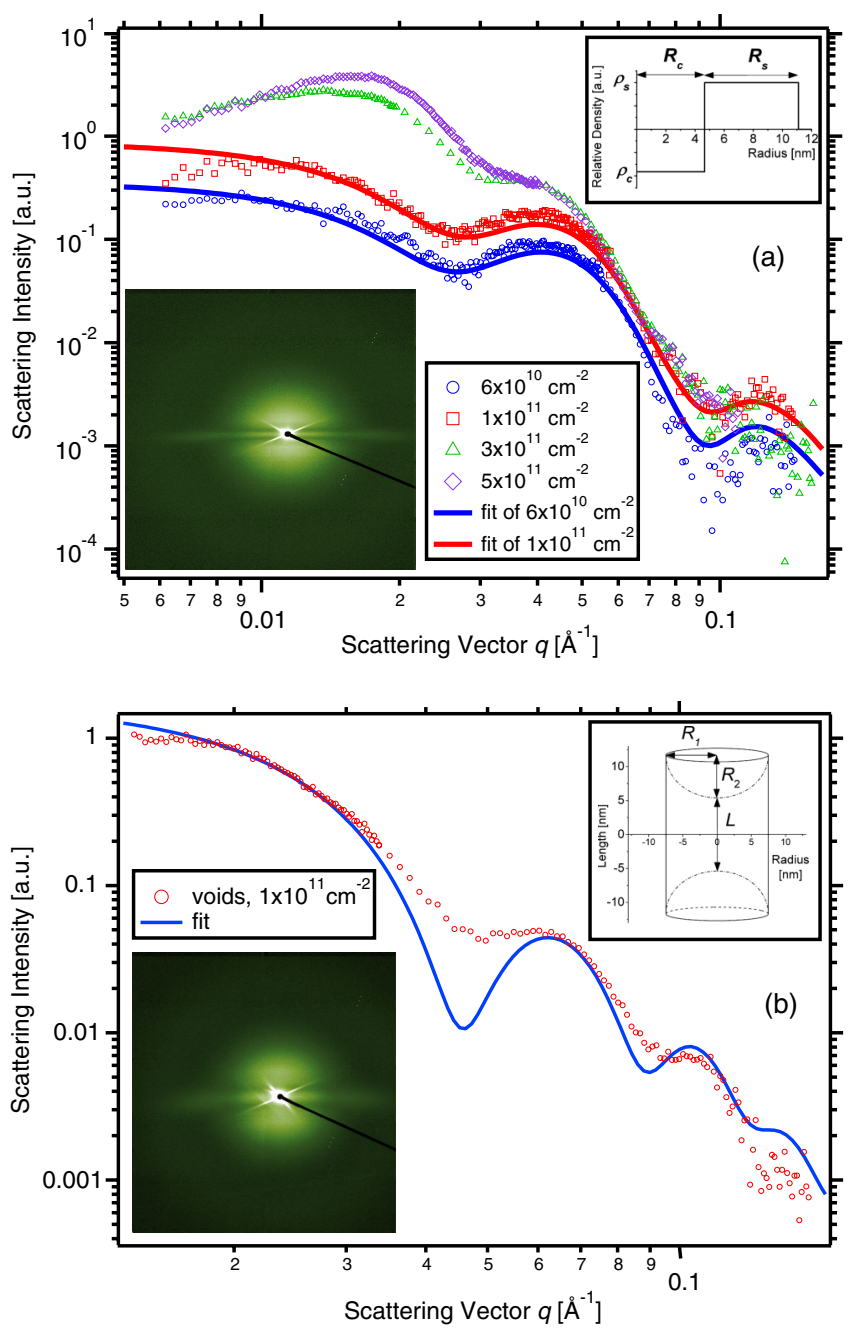

FIG. 1 (color online). (a) Background-subtracted SAXS intensity as a function of scattering vector for ion tracks including the detector image recorded with the surface normal at $10^{\circ}$ to the $\mathrm{X}$-ray direction (bottom left) and the calculated radial density distribution (top right). (b) Background-subtracted SAXS intensity as a function of scattering vector for voids including the detector image recorded with the surface normal at $5^{\circ}$ to the $\mathrm{x}$-ray direction (bottom left). The void geometry used for fitting (top right) was 10.6, 7.4, and $6.6 \mathrm{~nm}$ for $L, R_{1}$, and $R_{2}$, respectively.

vector $q$, isolated and background subtracted, is shown in Fig. 1 for two orientations. In both spectra, the scattering contributions from ion tracks and voids are apparent as horizontal streaks and mirrored hemispheres, respectively. Long, parallel cylindrical-like features (ion tracks) yield the streaks which are compressed in the detector image upon tilting. The voids responsible for the second scattering component were identifiable with TEM.

In Fig. 1(a), $I$ increases as a function of fluence consistent with an increase in the number of scattering centres, in this case ion tracks. As the fluence exceeds $10^{11} / \mathrm{cm}^{2}$, the well-defined oscillations wash out as ion-track overlap becomes more probable. Fitting the two lower fluences 
TABLE I. Ion-track structural parameters determined with SAXS. $R_{\text {core }}, R_{\text {shell }}$, and $R_{\text {total }}$ are the core radius, shell thickness, and total radius, respectively; $\sigma_{\text {core }}, \sigma_{\text {shell }}$, and $\sigma_{\text {total }}$ are the standard deviation of the Gaussian distributions used to account for the polydispersity of the core, shell, and total radii, respectively; $\Delta \rho_{\text {core }}$ and $\Delta \rho_{\text {shell }}$ are the change in density of the core and shell relative to the unirradiated matrix, respectively.

\begin{tabular}{lcccccc}
\hline \hline$R_{\text {core }}(\mathrm{nm})$ & $\sigma_{\text {core }}(\mathrm{nm})$ & $R_{\text {shell }}(\mathrm{nm})$ & $\sigma_{\text {shell }}(\mathrm{nm})$ & $R_{\text {total }}(\mathrm{nm})$ & $\sigma_{\text {total }}(\mathrm{nm})$ & $\Delta \rho_{\text {core }} / \Delta \rho_{\text {shell }}$ \\
\hline $4.7 \pm 0.1$ & 0.73 & $6.5 \pm 0.1$ & 1.01 & $11.1 \pm 0.2$ & 1.69 & $-0.91 \pm 0.14$ \\
\hline \hline
\end{tabular}

with a core-shell model yields the ion-track parameters given in Table I including a total track radius of $11.1 \pm$ $0.2 \mathrm{~nm}$. (The measured radius of an ion track is considered indicative of the maximum melt radius [12,24].) A negative value of the core-to-shell density ratio $\left(\Delta \rho_{\text {core }} / \Delta \rho_{\text {shell }}\right.$ where $\Delta \rho_{\text {core }}$ and $\Delta \rho_{\text {shell }}$ are the change in density of the core and shell relative to the unirradiated matrix, respectively) indicates core and shell have a density lower and higher, respectively, than the surrounding matrix (or vice versa). We suggest the shell is overdense, consistent with frozen-in radially outward material flow from the ion-track core following a thermal spike. The relative radial density distribution across the ion track derived from SAXS measurements is shown in the Fig. 1(a) inset. This distribution, corroborated by simulations presented below, yields a net volume contraction that is accommodated by the creation of open volume.

Figure 1(b) shows the scattering contribution from the second component, fitted with the bow-tie shape shown in the inset. This choice of shape was governed by TEM observations shown in Fig. 2 which depict a void. TEM through-focus analysis confirmed these features are less dense than the matrix. The parallel sides of the void are aligned with the incident ion direction, suggesting their formation mechanisms are correlated. Table II lists the void parameters derived from SAXS and TEM measurements.

Insight into the ion-track and void formation processes was garnered from MD simulations (see Supplemental Information animation [14]). Using the SW potential, the solid-to-liquid phase transformation along the ion track

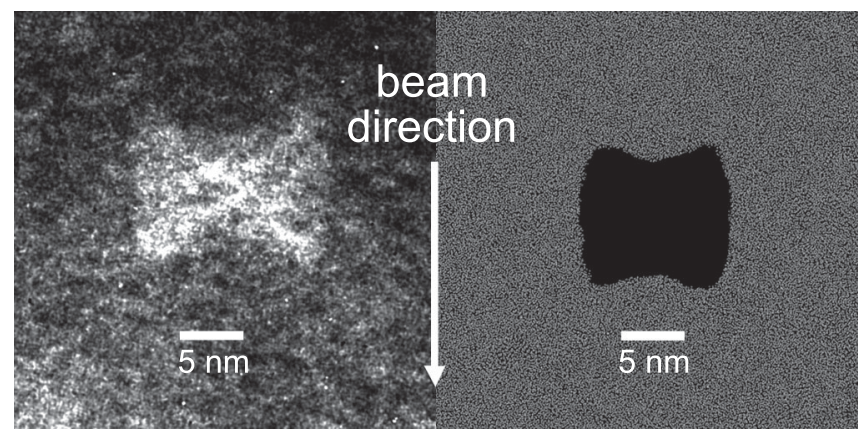

FIG. 2. XTEM image from $a$-Ge irradiated to a fluence of $6 \times 10^{10} / \mathrm{cm}^{2}$ (left) and MD simulation of the void geometry following resolidification (300 ps) (right). was apparent with MD. The density of the sixfoldcoordinated molten Ge exceeded that of the fourfoldcoordinated amorphous Ge, as expected [25]. Within the molten material, spherical voids appeared at $\sim 10$ ps followed by void agglomeration for surface-area minimization. Prior to resolidification, the void shape was prolate ellipsoid with the major axis aligned with the incident ion direction. The minor axis equalled $\sim 60 \%$ of the molten ion-track width. For our ion energy deposition parameters, void formation was not apparent using the Tersoff potential.

While the SW potential effectively yielded the highdensity liquid phase upon melting, this density was retained upon resolidification for the simulation of the ion track and a bulk cell of liquid $\mathrm{Ge}$ quenched at $3 \times 10^{13} \mathrm{~K} / \mathrm{s}$, a rate comparable to that for the ion track. In contrast, the Tersoff potential reproduced the lowdensity amorphous solid upon quenching of the bulk cell of liquid Ge. We thus implemented the Tersoff potential at $100 \mathrm{ps}$ for the MD simulation of ion-track formation, following which the transformation from high-density liquid to low-density solid was observable upon cooling.

The validity of changing potentials mid-simulation is demonstrated with Fig. 2, which shows the MD simulation of the void after resolidification of the molten ion track (300 ps total). The similarity between experiment and simulation is remarkable. While spherical voids form as a consequence of the volume contraction induced by the low-density solid to high-density liquid phase transformation, the peculiar shape of the voids upon resolidification is the result of radially inward cooling. As the molten ion track progressively solidifies from the maximum melt radius toward the ion-track core, the expanding volume of the solidified material compresses the residual molten material necessitating axial motion toward the void. The innermost volume experiences the greatest effect and a bow-tie shaped void results.

TABLE II. Void structural parameters determined with SAXS, XTEM, and MD. XTEM and MD values are estimated from very few occurrences.

\begin{tabular}{lcc}
\hline \hline & Radial Width $(\mathrm{nm})$ & Length $(\mathrm{nm})$ \\
\hline SAXS & $\sim 7$ & $\sim 24$ \\
XTEM & $\sim 7$ & $\sim 10-20$ \\
MD & $\sim 6$ & $\sim 10$ \\
\hline \hline
\end{tabular}



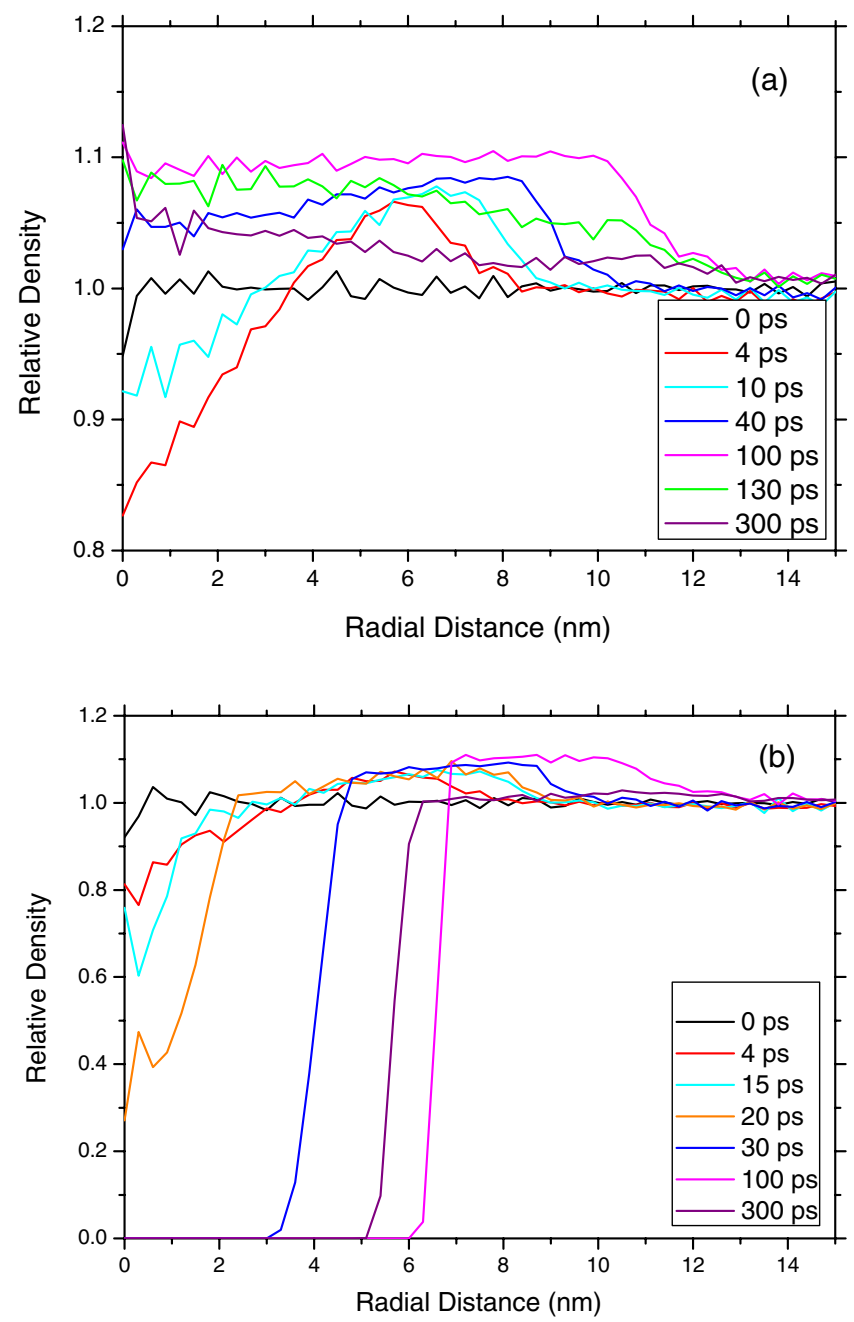

FIG. 3 (color online). MD simulation of the time-dependent radial density distribution across an (a) ion track and (b) void. In (a), the reduction in density at $\sim 10 \mathrm{~nm}$ radial distance for times $100-130$ ps is an artifact from changing the potential at $100 \mathrm{ps}$.

Figure 3 shows time-dependent MD results of the relative radial density across (a) an ion track (between voids) and (b) a void, respectively. Across the ion track, the development of the core-shell structure is rapid with the greatest density ratio achieved $\sim 4$ ps after the ion energy deposition. At the same time, the maximum temperature was achieved within the ion track $(\sim 4600 \mathrm{~K})$, a temperature beyond that required for boiling (3093 K) [26]). The maximum melt radius $(\sim 11 \mathrm{~nm})$ was reached at $100 \mathrm{ps}$ and the presence of the high-density liquid phase spanning the ion track is readily apparent. Thereafter, the ion-track density distribution relaxes toward the preirradiation, low-density amorphous phase. MD yielded no evidence of crystallization within the ion track upon resolidification, consistent with XTEM observations. Both results contradict those of Furuno et al. [9] potentially due to the influence of the free surface in Ref. [9]. Across the void, formation begins in the vapor phase
(10 ps) but evolves fully in the liquid phase ( $>25 \mathrm{ps})$. The maximum radii of the void and molten track $(\sim 6.5$ and $\sim 11 \mathrm{~nm}$, respectively) are achieved at the same time (100 ps) again suggesting their formation is correlated. Relaxation yields a slight narrowing of the void with the radial density distribution constant for $\geq 200 \mathrm{ps}$. The entire cell has cooled to below the melting point of $a$-Ge (965 K [27]) by 300 ps.

We propose the following scenario for ion-track and void formation in $a$-Ge. For our irradiation conditions, the energy transferred from the electronic to lattice subsystems is sufficient to induce melting and the formation of a molten cylinder of radius $\sim 11 \mathrm{~nm}$. For elemental Ge, the liquid-phase density exceeds that of the solid and this increase in density necessitates a volume contraction within the ion track upon melting. Void formation begins in the vapor phase but clearly evolves in the liquid phase as shown from our MD simulations. As the lattice cools, our SAXS results show remnants of a core-shell structure are quenched into the ion track and radially inward resolidification yields bow-tie shaped voids.

We now calculate the number of voids per unit track length using two approaches. For the first, we estimate an ion track contains $2.8 \pm 0.5$ voids $/ \mu \mathrm{m}$ based on the amplitude of the SAXS spectrum. For the second, we approximate the void as a cylinder (13-nm wide and 15-nm long from XTEM) and calculate $99 \%$ surface coverage is achieved with a fluence of $\sim 3.5 \times 10^{12} / \mathrm{cm}^{2}$ (using an exponential overlap model (fractional coverage $=$ $1-\exp \left(-\pi R_{v}^{2} \phi\right)$ with $R_{v}$ the void radius and $\phi$ the fluence). Using a void length of $15 \mathrm{~nm}$ as above and the irradiation-induced swelling data for $a-\mathrm{Ge}$ from Steinbach et al. [6] (derived with conditions identical to those in this report), yields a second, independent estimate of $\sim 2.1$ voids $/ \mu \mathrm{m}$ in an ion track. (A third estimate from the MD simulation would clearly yield an erroneously high value given the limited length $(61 \mathrm{~nm})$ of the simulation cell. Our use of MD was to identify radial density distributions and the void formation mechanism.) This leads us to suggest that the bow-tie shaped features apparent in our XTEM image of Fig. 2 not only result from the contraction of the molten volume within an ion track but, furthermore, they represent the embryonic precursors to the porous structure induced in $a$-Ge by SHII.

In summary, we have identified and characterized ion tracks and voids in $a$-Ge, thus demonstrating ion-track formation in an amorphous semiconductor. SAXS measurements showed the ion track was comprised of a coreshell structure consistent with quenched-in material flow, the latter resulting from a thermal spike and emanating radially outward. MD simulations, using a MC TTM approach as input, showed not only that a core-shell structure is present in the early stages of ion-track formation but remarkably reproduced the void geometry apparent from XTEM observations. The evolution of the void size and 
shape was a natural consequence of the density difference between solid and liquid Ge.

This work was financially supported by the Australian Research Council, the Academy of Finland, the IT Centre for Science CSC (Finland) and the Deutsche Forschungsgemeinschaft. Parts of this research were undertaken at the Canberra node of the Australian National Fabrication Facility, the ANU Heavy-Ion Accelerator Facility (HIAF) and the SAXS/WAXS beamline at the Australian Synchrotron, Victoria, Australia. We thank the staff of the ANU HIAF for technical support and J. Fitz Gerald and D. J. Llewellyn for assistance with the XTEM measurements.

*Corresponding author. mark.ridgway@anu.edu.au

Present address: Instituto de Física, Universidade Federal do Rio Grande do Sul, Porto Alegre, Brasil.

${ }^{\ddagger}$ Present address: Australian Research Council, Canberra, Australia.

${ }^{\S}$ Present address: Donostia International Physics Center, San Sebastian, Spain.

"Present address: Center for Free Electron Laser Science, DESY, Hamburg, Germany.

[1] G. A. Wagner and P. Van den Haute, Fission-Track Dating (Kluwer, Dordrecht, 1992).

[2] D. Kanjilal, Curr. Sci. 80, 1560 (2001).

[3] P. Apel, Nucl. Instrum. Methods Phys. Res., Sect. B 208, 11 (2003).

[4] W. Wesch, A. Kamarou, and E. Wendler, Nucl. Instrum. Methods Phys. Res., Sect. B 225, 111 (2004).

[5] A. Colder, O. Marty, B. Canut, M. Levalois, P. Marie, X. Portier, S. M. M. Ramos, and M. Toulemonde, Nucl. Instrum. Methods Phys. Res., Sect. B 174, 491 (2001).

[6] T. Steinbach, C. S. Schnohr, W. Wesch, P. Kluth, R. Giulian, L. L. Araujo, D. J. Sprouster, and M.C. Ridgway, Phys. Rev. B 83, 054113 (2011).

[7] W. Wesch, C.S. Schnohr, P. Kluth, Z.S. Hussain, L. L. Araujo, R. Giulian, D. J. Sprouster, A. P. Byrne, and M. C. Ridgway, J. Phys. D 42, 115402 (2009).

[8] K. Gartner, J. Johrens, T. Steinbach, C. S. Schnohr, M. C. Ridgway, and W. Wesch, Phys. Rev. B 83, 224106 (2011).
[9] S. Furuno, H. Otsu, K. Hojou, and K. Izui, Nucl. Instrum. Methods Phys. Res., Sect. B 107, 223 (1996).

[10] M. Karlusic, S. Akcöltekin, O. Osmani, I. Monnet, H. Lebius, M. Jaksic, and M. Schleberger, New J. Phys. 12, 2345 (2010).

[11] A. Hedler, W. Wesch, and S. Klaumunzer, Nat. Mater. 3, 804 (2004).

[12] P. Kluth, C. S. Schnohr, O. H. Pakarinen, F. Djurabekova, D. J. Sprouster, R. Giulian, M. C. Ridgway, A. P. Byrne, C. Trautmann, D. J. Cookson, K. Nordlund, and M. Toulemonde, Phys. Rev. Lett. 101, 175503 (2008).

[13] J. F. Ziegler, J. P. Biersack, and U. Littmark, The Stopping and Range of Ions in Solids (Pergamon, New York, 1985).

[14] See Supplemental Material at http://link.aps.org/ supplemental/10.1103/PhysRevLett.110.245502 for details on the theoretical approach, MD animation, sample preparation and characterisation, and SAXS data analysis.

[15] N. A. Medvedev, A. E. Volkov, N. S. Shcheblanov, and B. Rethfeld, Phys. Rev. B 82, 125425 (2010).

[16] M. I. Kaganov, I. M. Lifshitz, and L. V. Tanatarov, Sov. Phys. JETP 4, 173 (1957).

[17] S. I. Anisimov, B. L. Kapeliovich, and T. L. Perel'man, Sov. Phys. JETP 39, 375 (1974).

[18] M. Toulemonde, C. Dufour, and E. Paumier, Phys. Rev. B 46, 14362 (1992).

[19] H. O. Jeschke, M.S. Diakhate, and M.E. Garcia, Appl. Phys. A 96, 33 (2009).

[20] K. Nordlund, M. Ghaly, R. S. Averback, M. Caturla, T. Diaz de la Rubia, and J. Tarus, Phys. Rev. B 57, 7556 (1998).

[21] M. Posselt and A. Gabriel, Phys. Rev. B 80, 045202 (2009).

[22] J. Tersoff, Phys. Rev. B 39, 5566 (1989).

[23] F. Wooten, K. Winer, and D. Wearie, Phys. Rev. Lett. 54, 1392 (1985).

[24] A. Chettah, H. Kucal, Z. G. Wang, M. Kac, A. Meftah, and M. Toulemonde, Nucl. Instrum. Methods Phys. Res., Sect. B 267, 2719 (2009).

[25] J. Koga and T. Yamaguchi, Phys. Chem. Glasses 46, 425 (2005).

[26] www.webelements.com

[27] E. P. Donovan, F. Spaepen, D. Turnbull, J. M. Poate, and D. C. Jacobson, J. Appl. Phys. 57, 1795 (1985). 